\title{
Évaluation, motivation et formation
}

\section{Patrick Doucet}

\section{OpenEdition \\ Journals}

Édition électronique

URL : http://journals.openedition.org/asp/3156

DOI : 10.4000/asp.3156

ISSN : 2108-6354

\section{Éditeur}

Groupe d'étude et de recherche en anglais de spécialité

\section{Édition imprimée}

Date de publication : 1 décembre 1997

Pagination : 255-270

ISSN : 1246-8185

\section{Référence électronique}

Patrick Doucet, «Évaluation, motivation et formation », ASp [En ligne], 15-18| 1997, mis en ligne le 07 novembre 2012, consulté le 01 mai 2019. URL : http://journals.openedition.org/asp/3156 ; DOI :

$10.4000 / a s p .3156$

Ce document a été généré automatiquement le 1 mai 2019.

Tous droits réservés 


\title{
Évaluation, motivation et formation
}

\author{
Patrick Doucet
}

1 Cet article vise à rendre compte de deux expériences menées à l'UFR Sciences de l'Université de Poitiers, l'une en DEUG MIAS, en 1991-92, l'autre à l'IUP Génie des Systèmes Industriels, en 1993-94 et 1994-95.

2 Cette communication se situe dans le domaine général de la didactique des langues étrangères. Le rôle du didacticien dans ce cadre est d'intervenir sur une formation, en fonction des paramètres de l'apprentissage d'une part et des paramètres de l'enseignement d'autre part, dans le but de chercher des solutions de compromis à des problèmes de terrain ${ }^{1}$. En l'occurrence, le point de départ fut un problème d'évaluation : il s'agissait de répondre à une demande institutionnelle (examen, et notation) dans une formation où le faible nombre d'heures allié au grand nombre d'étudiants par groupe ne permettait pas un progrès linguistique mesurable. La question qui se posait était donc celle de la fonction de l'évaluation dans un tel cadre.

3 Le titre même de la communication oriente nos préoccupations vers la notion d'évaluation formative. Mais le rapport que nous envisageons est plus complexe : il s'agira de réconcilier des exigences d'évaluation en apparence contradictoires: celles de l'apprenant, celles de l'institution, et celles de l'enseignant. Il s'agit de trouver un moyen terme entre évaluation formative et évaluation normative.

4 C'est délibérément que le terme d'évaluation a été placé en premier, car l'évaluation est véritablement le moteur de la démarche adoptée. Nous proposons d'exposer comment les conditions peuvent être réunies pour que l'évaluation soit le ressort de la motivation des apprenants, influençant par là même la formation qui précède, qu'il s'agisse des activités proposées ou de l'investissement des apprenants dans la formation. Le choix du type d'évaluation, des contenus et des critères d'évaluation conditionne le reste de la démarche.

5 Avant de rendre compte des expériences, il convient dans un premier temps de délimiter brièvement le cadre théorique de référence. En effet, les termes d'évaluation, de motivation et de formation sont utilisés dans tellement de contextes différents qu'il est nécessaire de préciser par quelques définitions le sens des termes qui vont être employés. 


\section{Cadre théorique}

\section{Formation}

6 Le domaine de formation qui nous préoccupe est l'apprentissage de l'anglais langue étrangère en milieu institutionnel. Le cadre privilégié dans le cas de la Faculté des Sciences est le suivant :

7 - conception de la langue: la langue est conçue principalement comme moyen de communication et d'interaction.

8 - conception de la culture : celle-ci est conçue comme ce qui se réalise dans le comportement quotidien des natifs.

9 - conception de l'apprentissage: cette dernière est essentiellement cognitive interactionniste. L'appropriation d'une langue étrangère est vue comme le développement d'un processus cognitif et affectif complexe dépendant de variables internes (individuelles) et externes (rôle de l'input [Ellis 94]; et plus particulièrement de l'input négocié) suivant dans une large mesure son propre cours. Les facteurs considérés comme cruciaux sont l'attitude; (orientation sur un continuum ségrégatif/intégratif [Meisel 1980, 1981; Gardner \& McIntyre 1991]) et la motivation (dynamique, instrumentale et cognitive). Ils sont envisagés dans le rapport qu'ils peuvent entretenir avec la prise en charge progressive de l'apprentissage par l'apprenant lui-même. Une démarche heuristique d'apprentissage est encouragée.

\section{Compétence de communication}

10 Il n'y a pas actuellement consensus dans le domaine de l'acquisition des langues secondes sur un modèle de compétence de communication, et aucun des modèles existants n'est pleinement satisfaisant. Si nous nous sommes basés sur le modèle de Canale et Swain ${ }^{2}$ en quatre composantes, c'est qu'il a semblé plus opérationnalisable sur le terrain. Ladéfinition opératoire renvoie à la compétence de communication mesurée par un test d'interaction orale en binôme. Dans le cadre des étudiants du DEUG MIAS, nous avons mis l'accent sur la transmission du sens littéral des énoncés. L'insistance est sur les principes d'économie et d'efficacité du langage, c'est-à-dire que nous encourageons une utilisation claire et sans effort de ses ressources en vue de transmettre du sens, et ce quelles que soient ces ressources (verbales et non verbales). Le recours à des stratégies de communication est une partie intégrante de ce dispositif.

\section{Motivation}

11 Parmi les paramètres influant sur l'apprentissage, il est établi que l'attitude et la motivation jouent le rôle le plus constant ${ }^{3}$. Par motivation instrumentale, nous entendons le désir d'apprendre en fonction d'une utilisation projetée de la langue à plus ou moins long terme. Cette motivation ne joue pas si les besoins sont perçus comme trop éloignés. Il s'agit dans ces cas-là de créer des besoins relais (donc artificiels) à court/moyen terme (comme la réussite à l'examen). 
12 La motivation cognitive en tant qu'intérêt intrinsèque est, selon Keller (1984), " une réponse positive à des stimuli basés sur des structures cognitives existantes, de telle sorte que la curiosité des apprenants soit éveillée et maintenue ».

13 La motivation peut venir de ce que l'apprenant a des occasions de communiquer; elle peut également venir d'une participation graduelle à la prise de décision dans le contexte de l'apprentissage (Holec).

\section{Évaluation} comportements qu'il peut produire. Il faut s'assurer que l'on crée des conditions qui permettent à cet échantillon d'être le plus représentatif possible de ce que l'apprenant est capable de faire dans une situation donnée. Un concepteur de test doit réfléchir aux conditions d'optimisation de la performance. Ceci ne revient cependant pas à dire qu'il ne doit pas y avoir de contraintes. Dans la mesure où certaines contraintes sont difficilement contournables, en milieu institutionnel notamment (lieu, heure, taille des groupes...) et où d'autres sont parfois souhaitables (performance en temps réel par exemple), c'est donc un compromis qu'il faut rechercher.

17 La définition que nous choisirons pour l'évaluation est la collecte systématique d'informations dans le but de prendre une décision. Le type de décision finale conditionne la nature de l'évaluation. Il s'agit ensuite de sélectionner des critères pertinents que l'on envisage de mettre en relation avec un système de référence. À l'intérieur d'une production, on effectue donc une sélection d'informations: les informations sélectionnées sont les indicateurs de performance dont il faut vérifier l'adéquation avec les critères qui en constituent la pertinence par rapport aux objectifs. Il y a donc recherche des significations du degré d'adéquation entre les indicateurs de performance et le système de référence. Cette recherche repose sur une comparaison.

(Morrow 1979) préférable d'évaluer la compétence de communication au moyen de tests directs, critériés, où l'apprenant doit démontrer son savoir-faire dans l'accomplissement d'une tâche de communication. Les tests vont différer selon les comportements évalués.

19 Si l'on admet que la compétence de communication est tributaire des capacités individuelles de traitement de l'information, il est logique de penser que le comportement langagier démontré (l'utilisation de la langue en situation) dépendra du type et de la complexité des données à traiter, de la quantité de données à traiter et du temps dont l'apprenant dispose pour agir ou réagir. Si l'on s'en tient à la distinction entre traitement contrôlé et automatisé (McLaughlin 1987) des données par l'apprenant, il est possible de supposer que le mode écrit favorisera davantage l'aspect contrôlé du 
traitement, grâce à la possibilité de faire des retours en arrière (en réception comme en production) ainsi que des corrections de sa production. Par contre, le mode oral s'accompagne d'une tension communicative, due à la situation et à l'intention de communication; il permet donc de rendre compte d'une utilisation plus spontanée de la langue. L'interaction orale, notamment, réduit au minimum le temps de traitement de l'information ; les ressources disponibles ne sont alors pas les mêmes que lorsqu'un temps de préparation est fourni ; elles sont très probablement moins importantes. L'apprenant doit faire appel en priorité aux processus automatisés et pallier les problèmes d'actualisation des connaissances par un recours à des stratégies de communication.

Un test valide de la compétence de communication orale demande une performance en temps réel où l'apprenant aura la possibilité de démontrer sa capacité à transmettre un contenu et à négocier du sens;, en vue d'atteindre un objectif communicatif.

Dans un cadre institutionnel, il convient de distinguer finalités de l'apprentissage et finalités de l'enseignement. Deux séries distinctes de paramètres jouent (Narcy 1992). D'un côté se trouve un individu, de l'autre une institution. D'un côté, il faut faire face à une demande sociale, de l'autre à une contrainte institutionnelle. L'enseignant de terrain est au point de rencontre de ces deux exigences, dans une position qui peut être difficile à tenir.

Les attentes de l'institution en matière d'évaluation sont avant tout normatives, éventuellement sommatives. Les attentes du didacticien sont avant tout formatives. Il est remarquable de constater que si la didactique place l'apprenant au centre des préoccupations en ce qui concerne l'apprentissage, les pratiques évaluatives ne sont souvent pas en cohérence avec cette démarche, et placent fréquemment l'institution au centre. La question qui se pose est donc de chercher les moyens de réconcilier des exigences qui peuvent paraitre contradictoires: comment proposer un type d'évaluation qui soit acceptable par toutes les parties, c'est-à-dire qui fournisse des informations qui satisfassent toutes les parties? Cette question fut le point de départ des expériences menées à l'UFR Sciences de Poitiers.

Dans ce cadre théorique, il faut également citer ce que l'on nomme l'effet de reflux de l'évaluation : il s'agit de l'influence qu'a un test sur la formation qui précède. Cet effet, qui se situe au coeur des relations qui existent entre évaluation et formation, est mentionné dans la plupart des ouvrages sur l'évaluation, mais aucune étude n'avait jusqu'ici tenté de le mesurer par l'expérience. C'est ce que nous nous sommes proposés de faire à la Faculté des Sciences.

Dans la mesure où l'effet de reflux (sur l'enseignement, mais aussi individuellement sur l'apprentissage) ne peut pas être négligé, il faut s'assurer que son influence soit positive. C'est-à-dire que le type d'activité proposée lors des tests est de nature à favoriser l'apprentissage. Les tests doivent d'autre part être crédibles d'abord auprès des apprenants qui auront à s'impliquer dans la formation envisagée. Cette crédibilité renforce la validité d'ensemble des tests que l'on pourra proposer aux apprenants. 


\section{Expériences}

\section{Expérience en DEUG}

\section{Un public spécifique}

Le public concerné est celui de certaines filières de la Faculté des Sciences de Poitiers, que l'on peut caractériser par une démotivation par rapport à l'apprentissage de l'anglais, une dévalorisation de cette matière dans le cursus, et des besoins éloignés. Nombre de ces étudiants avaient abandonné l'idée de pouvoir un jour faire des progrès en anglais. Il est probable qu'en apprentissage d'une langue étrangère en milieu institutionnel l'apprenant qui a perdu confiance en soi, en ses capacités de réussir, risque de ne s'investir que très légèrement dans la démarche d'apprentissage. C'est encore plus vrai s'il n'a pas confiance en l'institution pour lui donner les moyens de progresser. Les procédures d'évaluation formative jouent ici un rôle non négligeable à tous les niveaux.

Il semble logique de penser qu'une motivation instrumentale ne jouera pas si les besoins perçus sont trop éloignés; et qu'il y a besoin de création de ce que J.P. Narcy appelle des besoins relais :

Les besoins relais sont des besoins langagiers, créés artificiellement pour compenser les manques de l'apprentissage, quand les besoins réels sont éloignés.

$(1990: 227)$

Lorsque les étudiants sont démotivés, on est amené à repenser les modes d'exposition à la langue. Un moyen de vaincre leur réticence est de proposer un cadre de formation où une évaluation crédible leur permet de mesurer des progrès, afin de recréer une dynamique d'apprentissage par un investissement personnel dans la formation.

\section{Action envisagée}

Il s'est agi de la conception d'un test crédible, apte à inciter des apprenants démotivés à s'investir dans leur formation, tout en étant susceptible de générer des situations de formation aptes à favoriser l'apprentissage. Les facteurs mis en avant en faveur de l'établissement d'un test d'interaction orale furent la volonté délibérée de prendre en compte la compétence stratégique, et la recherche d'une influence sur la motivation des apprenants, motivation non seulement inhérente à la tâche elle-même, mais aussi dynamique, en fonction du test à venir. Il s'agissait en effet de réconcilier certains publics avec l'apprentissage de l'anglais en choisissant un nouvel angle d'approche, celui de la recherche de l'atteinte d'objectifs communicatifs, modestes dans un premier temps, mais réels. On proposait aux étudiants de détourner pour un temps la focalisation de la forme vers le sens.

Le format choisi fut l'interaction orale en binôme (pair à pair). Deux tâches successives étaient proposées aux candidats, l'une basée sur un écart d'information, l'autre sur une différence d'opinions. La durée des interactions était fixée à 10 minutes.

L'hypothèse était de postuler l'existence d'un effet de reflux de l'évaluation sur la formation qui précède. Les conditions mises en place pour créer cet effet furent de faire passer le test par anticipation sous forme d'un pré-test en début de formation et de proposer lors de la formation un certain nombre d'activités « préparatoires » au test. 
31 Nous décrirons très brièvement les résultats de la première expérience dont la mise en place a déjà fait l'objet d'une communication ${ }^{6}$. Lors de cette expérience, les données furent obtenues par enregistrement de toute la population concernée (pré-test), sélection aléatoire d'un échantillon de 100 personnes représentatif de l'ensemble ${ }^{7}$, transcription des conversations, puis analyse et finalement étude statistique simple (distributions et corrélations). Il s'est agi de mesurer l'écart entre les performances entre le pré-test et le test.

L'analyse des performances au test a montré que les étudiants ont eu d'une façon générale une expression plus fluide, et que l'augmentation du débit, de pair avec le nombre réduit d'hésitations a permis des échanges plus fructueux qui se sont traduits par une augmentation des résultats en ce qui concerne le contenu des interactions. L'effet positif de la prise en compte du contenu dans les critères d'évaluation doit être souligné, car il rend des progrès facilement mesurables par l'apprenant lui-même. Il y a donc eu optimisation de la performance entre le pré-test et le test.

On pouvait supposer qu'un entraînement aux activités et donc une plus grande familiarité avec le format du test sont de nature à améliorer la performance, mais qu'en était-il de l'effet de reflux du test en tant que tel? Il convenait de vérifier si l'influence sur la motivation des apprenants avait été, comme on pouvait le supposer, de nature à changer leur investissement dans la formation.

C'est ce que l'on a essayé de vérifier dans une deuxième expérience, à l'IUP cette fois, en intégrant l'effet de reflux ${ }^{8}$ dans un dispositif de formation plus étendu où les apprenants sont avertis du type de test à venir et ont en plus le choix des activités d'apprentissage. Nous allions alors pouvoir constater si les activités choisies en priorité étaient en rapport avec l'examen final. Nous serions ainsi à même d'explorer plus avant les rapports qui existent entre évaluation et motivation.

\section{Expérience à l'IUP}

\section{Un public spécifique}

Les étudiants qui ont participé à l'expérience qui va être décrite constituaient la première promotion d'ingénieurs-maîtres formés à Poitiers. Rappelons la durée des enseignements d'anglais pour ces étudiants : 40 heures en IUP 2 ( 20 seulement pour la première partie de l'expérience, de février à mai 1994) et 25 heures en IUP 3 (deuxième partie de l'expérience, d'octobre 1994 à janvier 1995).

36 Ces étudiants n'étaient pas très motivés par une matière qu'ils considéraient comme peu importante dans leur spécialité, voire ennuyeuse. Ils n'étaient pas convaincus de l'efficacité de l'apprentissage en milieu institutionnel, même s'ils portaient personnellement une partie de la responsabilité de la situation où ils se trouvaient. Ils ne voyaient pas le lien entre les activités qu'ils avaient faites en classe par le passé et la réalité de l'utilisation de la langue.

\section{Action envisagée : des ateliers d'anglais}

La proposition qui fut faite aux étudiants d'IUP $2^{\mathrm{e}}$ année fut une expérience de travail en ateliers d'anglais: il leur était possible de programmer librement les activités qu'ils allaient pratiquer, à partir d'un «menu »" 
38 On cherchait à amener les étudiants à une plus grande prise en charge de leur apprentissage : il s'agissait de montrer à l'apprenant qu'il peut agir sur son apprentissage en le laissant libre de prendre certaines décisions par rapport à cet apprentissage.

L'objectif général de formation était post-institutionnel: créer des conditions qui permettent à l'apprenant de se donner les moyens de continuer à apprendre seul, une fois sorti de l'institution.

On cherchait à introduire plus de souplesse dans le fonctionnement (ne pas imposer à tous la même chose; donner la possibilité de travailler davantage ses points faibles ou perçus comme tels). Dans le même temps, il serait possible d'essayer d'individualiser davantage l'enseignement : se donner le temps d'aider davantage ceux qui en ont besoin, être plus disponible pour apporter une aide, et pour répondre aux demandes. À la fin de chaque séance de travail, les étudiants ont rempli des fiches d'auto-évaluation devant servir de base à de courts échanges avec l'enseignant pendant la classe, afin de réfléchir sur leur apprentissage de la langue.

41 Cette expérience fournissait la possibilité d'étudier l'effet de reflux de l'évaluation sur une période prolongée : il s'agissait de voir si, dès lors qu'ils avaient le choix des activités d'apprentissage, les apprenants allaient travailler en fonction de l'examen (motivation dynamique) ou en fonction d'objectifs de formation personnelle (motivation cognitive). L'examen du rapport évaluation/motivation; permettrait de voir si la notion de relais de motivation pouvait s'appliquer à l'évaluation.

Cette deuxième étude est qualitative. On a procédé par questionnaires ouverts dans un premier temps, puis fermés. Un des objectifs du premier questionnaire (figure 1) était donc de faire émerger certaines représentations des étudiants dans ce domaine, mais surtout de faire réfléchir les étudiants sur le domaine, de les amener à se poser des questions (statut de l'erreur, rôle de l'enseignant...), à formuler des hypothèses qu'ils devraient confirmer/infirmer ensuite et à remettre en cause leurs apprentissages précédents. En fait, ils ont, et ce n'est pas pour surprendre, éprouvé des difficultés à répondre à certaines questions. 
Quelles sont pour vous les façons les plus efficaces d'apprendre une langue étrangère?

Quelles tâches/activités d'apprentissage d'une langue vous semblent les meilleures? Quelles sont les raisons de vos choix?

Que peut faire un enseignant pour vous aider le mieux possible quand vous apprenez une langue étrangère?

Que peuvent faire les autres étudiants de votre groupe pour vous aider le mieux possible quand vous apprenez une langue étrangère?

Quel est le type de leçon d'anglais que vous préférez ? Quelles sont les raisons de votre choix ?

Quels avantages et quels inconvénients y a-t-il à apprendre une langue en classe? (par opposition à sur le tas, dans le pays, etc.)

Quelle aide les documents (audio/vidéo; écrits) et les feuilles de travail (questionnaires, exercices) vous apportent-ils principalement dans votre apprentissage d'une langue étrangère?

Quels sont les meilleurs types de documents/feuilles de travail pour apprendre une langue étrangère?

Pouvez-vous les décrire ? Pourquoi pensez-vous qu'ils soient plus appropriés ?

Quels sont les bons points et les points critiquables des documents/feuilles de travail que vous avez utilisé cette année ? Qu'est-ce qui leur manque à votre avis?

Quels changements souhaiteriez-vous y apporter?

adapté de Breen \& Candlin (1987)

Il ressort généralement du dépouillement des questionnaires une confusion entre objectif et façon d'apprendre (un moyen d'atteindre l'objectif), une confusion entre acquisition et apprentissage, et une confusion entre travail de la compréhension et contrôle de la compréhension (apprentissage vs évaluation). D'autre part, les étudiants sont pratiquement tous convaincus que c'est lors d'un séjour linguistique que l'on apprend l'anglais de la façon la plus efficace : l'anglais s'apprend « sur le tas » (bain linguistique).

Le défaut de motivation mentionné plus haut apparait clairement dans ce premier questionnaire :en ce qui concerne les avantages et les inconvénients de l'apprentissage en milieu institutionnel, il est fait référence à des inconvénients 68 fois, contre 38 fois seulement aux avantages ; c'est dire le scepticisme des étudiants dans ce domaine !

De la même façon que lors de l'expérience précédente, un pré-test d'interaction orale fut administré en début de formation et un test en fin de première année.

Un questionnaire de bilan (fermé) fut rempli suite à ce test.

Des réponses apportées à ce questionnaire dépendaient la poursuite ou l'arrêt de l'expérience, et dans l'éventualité d'une prolongation de l'expérience, les modifications qu'il serait possible d'apporter à la formule.

Nous ne rapporterons ici que les réponses portant sur les choix d'activités, dans le cadre de l'étude sur les rapports qui existent entre évaluation et motivation: on demandait entre autres aux étudiants de dire, a posteriori, quelles activités ${ }^{10}$ ils avaient choisies en priorité (figure 2). 
Figure 2. Ateliers d'anglais IUP

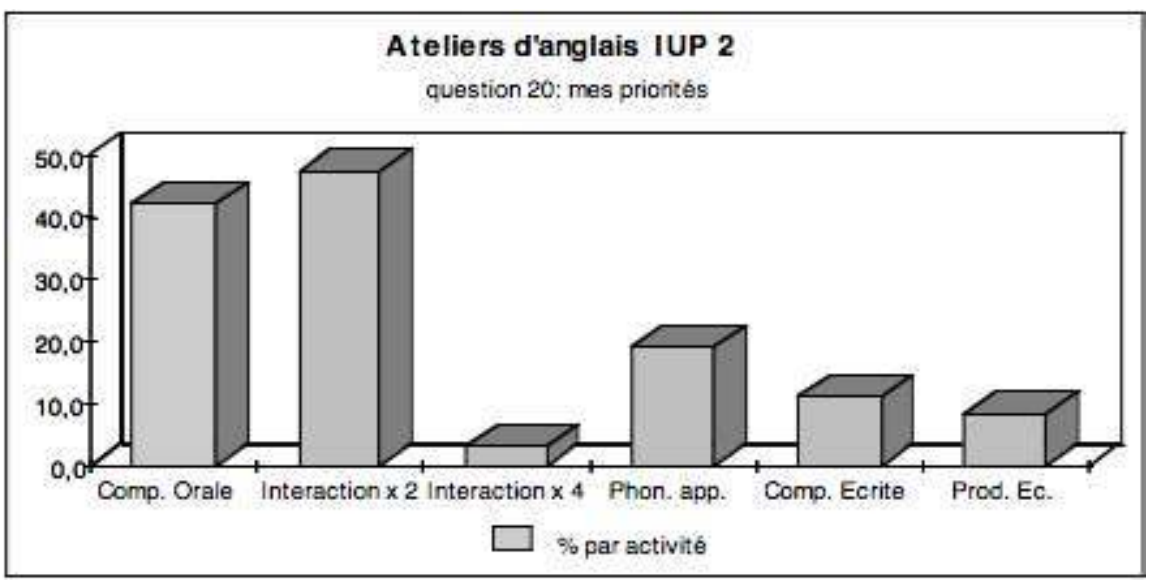

49 Il apparaît nettement qu'interaction et compréhension orales ont été privilégiées. Les activités les plus pratiquées pendant le cours furent donc essentiellement des activités en liaison avec l'objectif évaluatif spécifié.

Il était ensuite demandé aux étudiants de préciser ce qui avait motivé leurs choix.

Tableau 1. Réponses au questionnaire

\begin{tabular}{|l||l|}
\hline a) C'est une activité intéressante en soi & $97 \%$ \\
\hline b) C'est une activité que je réussis bien & $31 \%$ \\
\hline c) C'est une activité que je ne réussis pas bien & $33 \%$ \\
\hline d) C'est une activité qui demande peu d'effort & $11 \%$ \\
\hline \hline e) C'est une activité qui demande beaucoup d'effort & $58 \%$ \\
\hline f) Cela correspond à mon utilisation future de l'anglais & $78 \%$ \\
\hline 1 professionnellement & $47 \%$ \\
\hline f) Cela correspond à mon utilisation future de l'anglais & \\
\hline 2 dans ma vie privée & $78 \%$ \\
\hline \hline g) Cela correspond au type d'examen d'anglais que j'ai eu à passer & $69 \%$ \\
\hline \hline h) J'ai l'impression d'apprendre en faisant cette activité & $97 \%$ \\
\hline i) J'ai l'impression de progresser en faisant cette activité & $94 \%$ \\
\hline j) Cette activité me semble plus réaliste & $36 \%$ \\
\hline k) Cette activité me permet de mieux identifier mes points faibles & $75 \%$ \\
\hline l) Cette activité me permet de mieux identifier mes points forts & \\
\hline
\end{tabular}


5

On constate que la motivation ponctuelle repose avant tout sur l'intérêt des tâches proposées (motivation relais) (a) ; deuxièmement, qu'il faut une certaine crédibilité des tâches ; aux yeux des apprenants ( $\mathrm{f}$ et $\mathrm{j}$ ) dans le rapport à l'utilisation supposée/projetée de la langue en situation réelle ; troisièmement, qu'il faut une crédibilité des tâches en termes d'apprentissage de la langue (h, i, et v pour l'aspect diagnostic [auto-évaluation]). On constate enfin que la motivation dynamique est tout de même très présente $(\mathrm{g})$ à ce stade de l'expérience. L'évaluation finale est un aiguillon de la motivation. Nous y voyons une validation de l'effet de reflux; de l'évaluation dans la mesure où le choix des activités était libre.

Il convient donc d'insister sur l'effet de reflux de l'évaluation sur la démarche d'apprentissage. Il est possible d'affirmer qu'en ce qui concerne les étudiants de l'IUP le fait d'être prévenus à l'avance du type de test choisi a fortement contribué à orienter le choix des activités, en plus de l'investissement dans les activités. Il faut tout de même insister sur les autres facteurs présidant principalement à ce choix, à savoir, l'intérêt intrinsèque pour les activités, et le sentiment que l'accomplissement de ces activités était utile à leur apprentissage.

Les étudiants ont donc décidé de poursuivre l'expérience l'année suivante, et un test d'interaction orale en binôme fut à nouveau proposé à l'examen. Nous reproduisons ciaprès la partie du questionnaire final concernant la justification des choix d'activités pour cette deuxième année

Tableau 2. Réponses à la Question 6 « Comment J'ai choisi mes activités cette année » (39 réponses)

\begin{tabular}{|l|l|}
\hline a) C'est une activité intéressante en soi & $82 \%$ \\
\hline b) C'est une activité que je réussis bien & $18 \%$ \\
\hline \hline c) C'est une activité que je ne réussis pas bien & $38 \%$ \\
\hline d) C'est une activité qui demande peu d'effort & $7,7 \%$ \\
\hline e) C'est une activité qui demande beaucoup d'effort & $25.5 \%$ \\
\hline f) Cela correspond à mon utilisation future de l'anglais professionnellement/dans ma & $77 \%$ \\
vie privée & \\
\hline \hline g) Cela correspondait au type d'examen que j'aurais à passer & $15 \%$ \\
\hline h) J'ai l'impression d'apprendre en faisant cette activité & $66,6 \%$ \\
\hline \hline i) J'ai l'impression de progresser en faisant cette activité & $79,5 \%$ \\
\hline j) Cette activité me semble plus réaliste & $54 \%$ \\
\hline k) Cette activité me permet de mieux identifier mes points faibles & $54 \%$ \\
\hline l) Cette activité me permet de mieux identifier mes points forts & $7,7 \%$ \\
\hline
\end{tabular}


54

Invient de comparer ces résultats à ceux du questionnaire de fin de la première étape On constate que de la même façon qu'à mi-chemin de l'expérience, la motivation repose principalement sur l'activité elle-même (a). Les choix sont fortement motivés par des critères de progrès et d'apprentissage ( $h$ et $\mathrm{i}$ ) et à un moindre degré de diagnostic $(\mathrm{k})$. Les choix témoignent d'un investissement en vue d'une utilisation de la langue (f).

Mais à la différence du questionnaire de l'année précédente, le réalisme des tâches est beaucoup moins un critère de choix $(j)$; on ne peut plus parler de motivation dynamique créée par l'évaluation (g). Ceci témoigne d'une prise en charge de leur apprentissage par les apprenants.

On peut donc affirmer qu'il y a eu création d'une dynamique de formation, dynamique dont l'évaluation fut l'un des ressorts, avec une responsabilisation progressive de l'apprenant. On pourrait postuler l'existence d'un seuil de motivation, qui n'est pas encore totalement franchi lors de la première phase de l'expérience, mais qui l'est à la fin de cette deuxième phase. Au-delà de ce seuil, le statut de l'évaluation change.

57 Voici pour terminer les réponses aux questions sur l'évaluation de la motivation individuelle (voir tableau 3).

Tableau 3. Réponses aux questions sur l'évaluation de la motivation individuelle

\begin{tabular}{|l|l|l|l|l|l|}
\hline Par rapport à l'an dernier... & \multicolumn{2}{|l|}{38 réponses } & \multicolumn{2}{|l|}{} \\
\hline a) Je me sens plus motivé & \multicolumn{2}{|l|}{21} & & \\
\hline b) Je me sens moins motivé & 0 & & \\
\hline c) Ma motivation est la même & 17 & & & \\
\hline d) J'évalue ma motivation de 1 à $4(1=$ non motivé $)$ & score & 1 & 2 & 3 & 4 \\
\hline \hline & & 0 & 1 & 30 & 7 \\
\hline
\end{tabular}

Le fait remarquable est que cette motivation, égale ou supérieure à celle de l'année précédente, n'est plus d'ordre dynamique (cf. question $6 \mathrm{~g}$ citée plus haut), mais cognitif. On ne peut qu'espérer qu'une fois sortis de l'institution (quelques semaines plus tard) ces futurs ingénieurs-maîtres ont su se donner les moyens de continuer à apprendre seuls, en fonction de leurs besoins.

semblerait donc que ce que l'on appelle the washback effect of testing, l'effet (positif ou négatif) de reflux qu'a l'évaluation sur l'enseignement et l'apprentissage qui précède, soit seulement une étape : cela n'a pas joué lors de la deuxième série de cours (troisième année IUP). Mais tout semble indiquer que, pendant cette période, les étudiants ont pris conscience des enjeux et qu'une motivation cognitive; a remplacé une motivation dynamique, ce qui nous permettrait de dire que nos pratiques évaluatives ont pleinement joué leur rôle de relais de motivation. 


\section{Interaction entre évaluation, formation et motivation}

60 schématiser les rapports qui existent entre évaluation, formation et motivation; de la
façon suivante, sous forme d'une série d'effets (voir figure 3). Figure 3

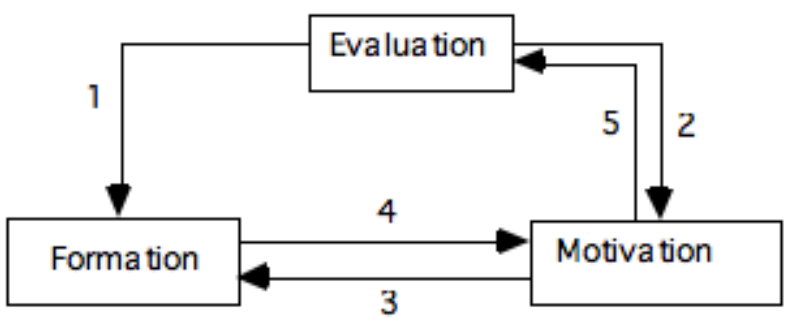

61

Dans ce schéma, nous prenons formation au sens restreint, c'est-à-dire ensemble des activités d'apprentissage à l'exclusion des bilans et tests, car il va sans dire que dans un contexte formatif il conviendrait d'inclure les pratiques évaluatives. Les différents chiffres correspondent aux différents effets.

Effet 1 : L'évaluation est déterminée en premier, en fonction de la nature de la décision que l'on souhaite prendre. Puis l'on conçoit le cours comme une "préparation » à l'examen, c'est-à-dire que les activités sont conçues en vue d'optimiser la performance des apprenants lors de cet examen. L'effet 1 s'applique au gestionnaire de la situation d'enseignement/apprentissage, qui peut être l'enseignant, ou l'apprenant lui-même.

Effet 2 : L'évaluation est présentée aux étudiants sous forme d'un pré-test crédible. Un bilan individuel a lieu permettant un positionnement personnel. Une motivation dynamique est créée.

l'évaluation change de nature en ce qui concerne l'apprenant. Cet enjeu, qui initialement se confondait avec l'épreuve elle-même, dépasse maintenant cette épreuve. L'examen n'est plus qu'une étape de régulation sur la voie d'une autonomie langagière, qui ne sera sans doute atteinte que lorsque l'apprenant sera sorti de l'institution. On se rend compte d'une part, que la régulation de l'apprentissage sera facilitée si l'apprenant est capable de s'auto-évaluer et, d'autre part, que la démarche proposée rend possible, voire facilite l'apprentissage de l'auto-évaluation.

67 Nos recherches sur l'effet de reflux portent à penser que si l'examen proposé aux apprenants ne correspond pas aux objectifs réels de l'institution, l'apprentissage se fera mal. Le fait que les nouvelles épreuves de langues étrangères des baccalauréats scientifiques ne comportent que des tests écrits est inquiétant dans la mesure où se creuse un fossé entre une demande sociale forte et une formation qui ne se donne pas les moyens d'y répondre. Si 
l'objectif à court terme des apprenants n'est autre que d'être reçu à l'examen (ce qui est souvent le cas) leur démarche d'apprentissage s'infléchira dans le sens d'une optimisation de leur performance au dit examen, au détriment éventuel des capacités non mesurées par les tests, principalement la capacité de s'exprimer et à négocier le sens oralement. Inversement l'apparition de diplômes tels que le DCL, qui tentent de mesurer la compétence de communication sous ses multiples formes est de nature à provoquer une remise en cause des pratiques pédagogiques et des démarches d'apprentissage en amont.

\section{Conclusion}

Dans le cadre de l'étude des rapports qui existent entre évaluation, formation et motivation, nous avons voulu démontrer une relation de causalité entre une pratique évaluative présentée par anticipation sous forme de pré-test et l'apprentissage qui précède, faisant par là même l'hypothèse de l'existence d'un effet de reflux positif ; qu'un test crédible peut avoir. Cet effet de reflux est souvent mentionné dans la recherche comme allant de soi (connaissance en tant que croyance), mais non mesuré. Il s'agit plutôt d'une variable que l'on cherche habituellement à contrôler: les chercheurs qui utilisent des tests pour vérifier l'incidence d'un type d'instruction sur l'acquisition essaient habituellement de neutraliser l'effet que la familiarisation avec le test a sur les performances. La démarche proposée ici est inverse : puisqu'on postulait l'existence d'un effet de reflux, il convenait de jouer sur cet effet pour optimiser la performance. Nous avons cherché à développer la familiarité de l'apprenant avec le type de test choisi. Nous nous sommes efforcés de vérifier par l'expérience le rôle de l'effet de reflux. Il semble que nous ayons obtenu des éléments qui permettent d'affirmer l'importance de ce phénomène dans le cadre de l'apprentissage d'une langue étrangère en milieu institutionnel.

Lors des échanges qui ont suivi cette communication, l'accent a été mis sur la difficulté d'expliciter de façon satisfaisante le rapport qui existe entre mesure quantitative et mesure qualitative lorsqu'il s'agit d'évaluer la compétence en langues.

71 Le DCL nouvellement créé tente d'apporter une réponse à ce dilemme qui se pose à chacun d'entre nous en séparant délibérément paramètres pragmatiques et paramètres linguistiques dans les grilles d'évaluation, et en leur affectant une pondération qui varie en fonction du degré d'opérationnalité démontré par les candidats ${ }^{11}$.

BIBLIOGRAPHIE

Bachman, L.F. 1990. Fundamental Considerations in Language Testing. Oxford : Oxford University Press.

Breen, M.P. and Candlin C.N. 1987. « Which materials?: a consumer's and designer's guide ». in Sheldon L.E. (eds). 
Canale, M. 1984. « Testing in a communicative approach ». In Jarvis, G. A. (ed.), Northeast Conference on the Teaching of Foreign Languages. The Challenge for Excellence in Foreign Language Education.

Dickinson, L. Self-instruction in Language Learning. Cambridge : Cambridge University Press.

Ellis, R. 1994. The Study of Second Language Acquisition. Oxford : Oxford University Press.

Isani, S. \& M. Herino. 1994. « La notion de scénario comme outil d'évaluation de la compétence en langue ». ASp 3, 107-109.

McLaughlin, B. 1988. Theories of Second Language Learning. Londres, New York : Edward Arnold.

Morrow, K. 1979. « Communicative language testing: Revolution or evolution ?». In Brumfit \& Johnson (eds), re-published in Issues in Language Testing in Alderson \& Hughes (eds), Oxford : Oxford University Press.

Narcy, J.P. 1990. Apprendre une langue étrangère. Paris : Les Éditions d'organisation.

Narcy, J.-P. 1994. « Autonomie, évolution ou révolution? ». Die Neueren Sprachen 93/5, 430-441.

Weir, C.J. 1993. Understanding and Developing Language Tests. Hemel Hempstead : Prentice Hall.

\section{NOTES}

1. Il s'agit toujours de partir d'une situation locale où l'on sera amené à privilégier certains paramètres.

2. Voici le modèle de Canale \& Swain en 4 composantes

compétence grammaticale (préférons le terme linguistique, cf. lexique) : Il s'agit du degré de maîtrise par l'apprenant du code linguistique, notamment: le vocabulaire, la prononciation, l'orthographe, la construction de mots et de phrases... Cette maîtrise est essentielle si les objectifs de l'apprenant sont élevés. Cette compétence englobe connaissances déclarative et procédurale. Il s'agit de comprendre et d'exprimer avec précision le sens littéral des énoncés.

compétence sociolinguistique :La compétence sociolinguistique permet la compréhension et l'expression correctes d'énoncés dans différents contextes sociolinguistiques. (Dans ce cas, le sens et la forme des énoncés sont adéquats). Elle renvoie au choix approprié de formesgrammaticales, de lexique, d'accent, en fonction du contexte sociolinguistique, pour faire passer des fonctions de communication spécifiques (persuader, décrire, raconter, ordonner...) Elle intègre le concept d'acte de parole, emprunté à la pragmatique. Elle dépend du rôle/du statut des participants, âge, rang social, cadre physique. Elle dépend également du sujet de la communication (des objectifs de l'interaction). Ceci conditionne une attitude à adopter, le choix d'un style, d'un registre.

compétence discursive:Il s'agit de lacapacité à combiner des idées pour obtenir cohésion de la forme et cohérence de la pensée. Elle permet de donner une unité à l'expression dans différents genres (narration, dissertation, compte-rendu scientifique, lettre d'affaires...). Cohésion: façon dont les énoncés sont liés structurellement pour faciliter l'interprétation d'un texte. (utilisation de mots de liaison, de pronoms, de synonymes, d'ellipses, de structures parallèles... qui permet la compréhension logique et chronologique d'un texte). Cohérence :relation entre les différents sens d'un texte, qu'ils soient littéraux, qu'ils aient une fonction sociale ou communicative.

compétence stratégique : Il s'agit de la capacité à utiliser des stratégies de communication : pour compenser les manques/vides dans la connaissance qu'a la personne du code (pour renforcer l'efficacité de la communication); pour compenser toute autre rupture/interruption de la communication (pannes de communication dues à des facteurs contraignants ou à une 
compétence insuffisante dans l'un ou l'autre des composant de la compétence de communication).

Canale (1983 : 339) complète sa définition pour intégrer la mise en valeur de l'effet rhétorique des énoncés, car il semble en effet que la compétence stratégique opère à tous les niveaux. C'est cependant le sens restreint qui sera privilégié dans les définitions opératoires lors des expériences décrites dans cet article.

3. La motivation instrumentale notamment semble être un meilleur prédicteur dans le contexte de l'apprentissage d'une langue étrangère que dans celui d'une langue seconde.

4. Nous devons répondre par la négative, car un échantillon ne peut représenter la variabilité ou la nature infinie de la langue.

5. Le DCL s'éloigne de la conception traditionnelle des trois champs d'application concernés par les langues étrangères (le général, le travail, les études). Un découpage différent est proposé : langue de communication usuelle, langue de communication à usage professionnel et langue de spécialisation. L'intersection entre ces trois ensembles est non vide, et c'est précisément dans le cadre de cette intersection que le DCL propose des tâches communicatives "transversales " (Isani \& Herino 1994).

6. XIII ${ }^{\mathrm{e}}$ Congrès du GERAS - Montpellier 1992, ASp 11993

7. L'échantillon du test fut choisi en fonction d'un éventail de performances stratégiques au prétest.

8. Rappelons l'hypothèse de l'effet de reflux: Les étudiants qui sont prévenus à l'avance du type d'examen choisi vont orienter leur démarche d'apprentissage dans ce sens, de façon volontaire. Ceci revient à faire de l'évaluation un moteur de la motivation, et par conséquent un moteur de l'apprentissage.

9. Il s'agit d'une mise en place progressive, avec un nombre limité d'activités possibles, mais toute suggestion ou demande spécifique des étudiants était prise en compte.

10. Successivement sur le graphique: compréhension orale, interaction orale en binôme, interaction en groupes de 4, phonétique appliquée, compréhension écrite, production écrite.

11. Ainsi, le premier degré du DCL (capacité opérationnelle minimale) est attribué uniquement en fonction des paramètres pragmatiques.

\section{RÉSUMÉS}

Dans le cadre d'un compte-rendu de deux expériences menées à l'Université de Poitiers (Faculté des Sciences, 1991-93 et 1993-95) sur des échantillons respectivement de 100 et de 44 étudiants, cet article vise à démontrer une relation de causalité entre une pratique évaluative présentée par anticipation sous forme de pré-test et l'apprentissage, postulant ainsi l'existence d'un effet de reflux positif qu'un test crédible peut avoir. Cet effet de reflux est souvent mentionné dans la recherche comme allant de soi, mais non mesuré. On insiste sur la notion de crédibilité des pratiques évaluatives aux yeux des apprenants: en l'occurrence, la compétence de communication est évaluée à l'aide d'un test d'interaction orale en binôme. On montre comment il est possible de créer des conditions pour que l'évaluation, conçue au départ comme ressort de la motivation, puisse en fin de compte n'être qu'une étape sur la voie d'une motivation intrinsèque, permettant la prise en charge de son apprentissage par l'apprenant lui-même. 
This article is an account of two experiments carried out in the Science faculty of Poitiers University (in 1991-93 and 1993-95) on two samples, one of 100 and the other of 44 students. A cause and effect relationship is established between language testing and language learning, when a pre-test of the same format is administered early in the course, thus ensuring positive washback. The washback effect of testing is often mentioned in research, but usually taken for granted and not measured. The author puts forward the face validity of testing where students are concerned. In the experiments, communicative competence is assessed through oral interaction in pairs. It can be seen that it is possible to create conditions whereby testing, originally designed to encourage student motivation, can actually be a step towards intrinsic motivation, a necessary condition for the learners to take on more responsibility for their own learning.

INDEX

Keywords : didactics, learning, motivation, testing, washback

Mots-clés : apprentissage, didactique, effet de reflux, évaluation, motivation

\section{AUTEUR}

\section{PATRICK DOUCET}

Patrick Doucet, auteur d'une thèse « L'évaluation en question » soutenue à l'Université de Technologie de Compiègne en 1996, est maître de conférences d'anglais à l'Université de Tours.

Patrick.Doucet@univ-poitiers.fr 